Article

\title{
Modeling and numerical simulation of a vegetation cover
}

\author{
Arturo Hidalgo $^{1, *}$ and Lourdes Tello ${ }^{2}$ \\ 1 Departamento de Ingeniería Geológica y Minera, ETS de Ingenieros de Minas y Energía, Center for \\ Computational Simulation, Universidad Politécnica de Madrid, Calle Ríos Rosas, 28003 Madrid, Spain \\ 2 Departamento de Matemática Aplicada, ETS de Arquitectura, Center for Computational Simulation, \\ Universidad Politécnica de Madrid, Av. Juan de Herrera, 28040 Madrid, Spain; 1.tello@upm.es \\ * Correspondence: arturo.hidalgo@upm.es
}

\begin{abstract}
The aim of this work is to introduce a mathematical model representing the evolution of the temperature in a vegetation cover and the ground underneath it. Vegetation, and its interaction with soil, plays a very important role in the protection of soil surface from the action of sun and precipitations. A reduction in the vegetated mass increase the risk of desertification, soil erosion or surface runoffs which which can give rise to soil loss and sediment retention. These processes can favour climate change and global warming, which are major concerns nowadays. The mathematical model presented takes into account the main processes involved in vegetation cover and the interaction with the soil, among which, we can mention the Leaf Area Index, which is a dimensionless quantity defined as the one-sided green leaf area per unit ground surface area, or albedo and co-albedo which are clearly influenced by the vegetation. It is also considered a nonlinear heat capacity in the soil which incorporates the latent heat of fusion, when the phase change takes place. The numerical technique used to solve the mathematical model is based on a finite volume scheme with Weighted Essentially Non Oscillatory technique for spatial reconstruction and the third order Runge-Kutta Total Variation Diminishing numerical scheme is used for time integration. Some numerical examples are solved to obtain the distribution of temperature both in the vegetation cover and the soil.
\end{abstract}

Keywords: vegetation cover; latent heat of fusion; climate model; climate change; energy balance models; finite volume method; WENO reconstruction; Runge-Kutta TVD

\section{Introduction}

Vegetation plays a very important role in the ecosystem of Earth since it is well related with climate and also has a great influence on environment, for instance to control soil erosion, dealing with soil formation or influencing the water cycle. Also, fires extinction can be helped by vegetation. Soil is a mixture of solid, liquid (water) and gas (air and water vapour). The thermal properties of the soil depend on the thermal properties of its constituents, their volume fractions, and how they are mixed. The heat capacity of a mixture of air, water and solids is the sum of the volume fractions, each multiplied by its heat capacity. Soil is a porous medium in which three phases can be distinguished: the solid matrix (minerals and organic material), the liquid phase (water) and the gaseous one (air and water vapour). Qualitatively, we can say that in unsaturated soils the heat will be transported in these three phases (see [1]). According to [2], a bare soil absorbs heat very fast, becoming hot during hot season and cold during the cold season. Vegetation acts as a thermal insulator and significantly affects the soil temperature. It does not allow the soil to become either too hot during the dry season or too 
cold during the rainy season. There are different pathways for heat transport in soils, namely radiation, conduction and convection. Heat transfer by radiation takes place between two bodies with different temperatures. This energy is emitted by an object in the form of photons or electromagnetic waves because of vibration and rotation of the atoms and the molecules. Conduction means the heat transfer when the objects are in physical contact, whereas convection represents the transfer of heat between the object and the environment. In [3] there is a study on the effects of vegetation and precipitation on the soil erosion. According to [4] vegetation affects erosion of the soil in the sense that it can be used to control erosion due to water. Also in [5] and references therein the relation between vegetation and soil loss due to runoffs is studied. Moreover the authors consider vertical vegetation structures, several kinds of plants and scale characteristics. In [6] it is introduced a conceptual model studying the relation between vegetation cover and land-cover. Vegetation cover can change in response to climate changes, so there is an important relationship between vegetation cover and global warming.

In [7] there is a mathematical model in partial differential equations (PDEs) is used to study landscape behaviours and dryland ecosystems. A study on the interactions between soil moisture and vegetation covers and its influence on soil temperatures when considering very water-limited environments is given in [8].

Soil temperature is relevant on soil properties and plant growth. In [2], and references therein, interesting aspects on the influence of soil temperature are described in detail, such as the influence of soil temperature on bioactivity, soil micro and macro-organisms and decomposition of organic matter. In addition, the authors describe other characteristics where soil temperature is very important. For instance, related to chemical properties of the soil, important phenomena are: cation exchange capacity (CEC), that is the total capacity of a soil to hold exchangeable cations, available phosphorous and soil $\mathrm{pH}$. Concerning the effect of the temperature on soil physical properties (see [2]) a dehydration process may happen, affecting soil structure. Furthermore thermal transformation of iron and aluminium oxides may produce changes in clay particles. Also, an increase in soil temperature can reduce water viscosity allowing it to percolate through the soil. Moreover, higher evaporation rates may arise, restricting the movement of water through the soil. Concerning soil aeration, carbon dioxide amount in the soil air can be affected by an increase in temperature. Finally, according to [2], soil temperature also has an influence on plant growth, such as nutrient and water uptake by plants or roots growth.

In this work we introduce and solve numerically a coupled model vegetation cover-soil, in order to analyze the thermal effects involved and the distribution of temperatures both in the vegetation cover and the soil. The model is based on ideas from a green roof model, proposed in [9,10], and on a climate model, see [11,12]. The numerical resolution is performed by means of a finite volume (FV) scheme with Weighted Essentially (WENO) reconstruction in space and a third order Runge-Kutta TVD (RK3-TVD) scheme for time discretization. The rest of the document is organized as follows. In the next section it is described the mathematical model introduced to represent the vegetation cover and its interaction with soil. The next section is devoted to a brief description of the numerical method used. Then, numerical examples are given. Finally some conclusions and discussion are given.

\section{The model}

In this approach a rectangular $2 D$ domain is considered, where the upper boundary represents the vegetation cover and the $2 D$ domain stands for the soil. In Figure 1 it is depicted the $2 D$ domain, where the upper side is the $1 D$ vegetation cover and the rest of the domain is the $2 D$ ground. A mixed layer inside the soil, represented by Dmix, has been introduced aimed to take into consideration the influence of the vegetation cover on the ground.

The diffusion equation representing the evolution of the temperature in the vegetation cover is given as

$$
C_{v} \frac{\partial T_{v}}{\partial t}-\frac{\partial}{\partial x}\left(K_{H 0} \frac{\partial T_{v}}{\partial x}\right)+K_{V} \frac{\partial T_{s}}{\partial n}=F_{v}
$$




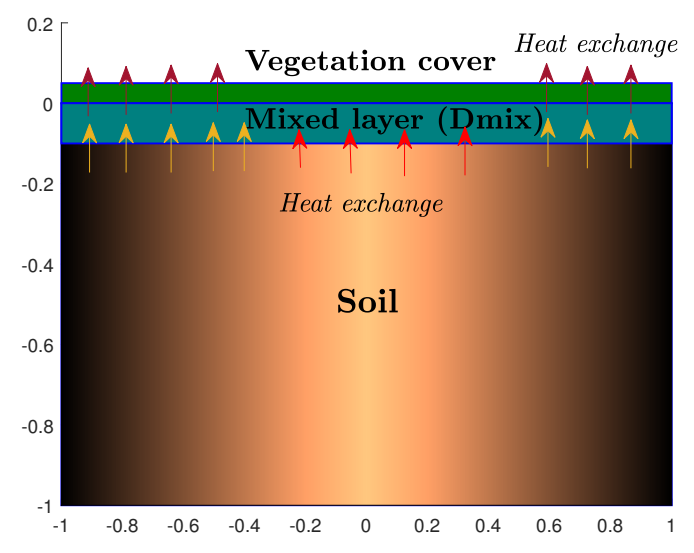

Figure 1. Domain considered in this model. The upper part is the $1 D$ vegetation cover, the $2 D$ region is the ground. The depth Dmix represents the mixed layer which is the part of the ground thermally influenced by the vegetation cover. Local reference coordinates $[-1,1] \times[-1,0]$ are used in the plot.

where $T_{v}=T_{v}(x, t)$ is the temperature in the vegetation cover, $T_{S}=T_{S}(x, z, t)$ is the temperature of the soil, $K_{H 0}$ is the heat conductivity in the vegetation cover, $K_{V}$ is the conductivity of the soil in vertical direction, $C_{v}$ the heat capacity of the vegetation cover, $x$ is the spatial coordinate, $t$ is time. The term $K_{V} \frac{\partial T_{s}}{\partial n}$ which considers the normal derivative of the soil temperature, represents the influence of the soil temperature onto the upper boundary where the vegetation is located. This term has been introduced to account for conduction phenomena from the ground to the vegetation cover.

This kind of dynamic and diffusive boundary condition appears also in many contexts, among them, we can mention a model arising in Biology $([13,14])$ for the analysis of inflammatory process in the artery wall and a climate model including surface-ocean interaction ([12]).

In (1) the source term is given by

$$
F_{v}=\sigma_{v}\left(R_{a v}+\epsilon_{v} I_{i r}-\epsilon_{v} \sigma T_{v}^{4}\right)+\frac{\sigma_{v} \epsilon_{s} \epsilon_{v} \sigma}{\epsilon_{l}}\left(T_{s}^{4}-T_{v}^{4}\right)+H_{v}+L_{v}
$$

where $H_{v}$ represents the sensible heat flux, which stands for the energy loss by the vegetation cover by heat transfer to the surrounding air and, according to $[9,15]$

$$
H_{v}=\left(e_{0}+1.1 L A I \rho_{a f} C_{a} C_{f} W_{a f}\right)\left(T_{a}-T_{v}\right),
$$

being $e_{0}$ the windless exchange coefficient which depends just on temperature difference, $L A I$ is the leaf area index, which is a dimensionless quantity which is defined as the one-sided green leaf area per unit ground surface area. The density of the foliage is represented by $\rho_{a f}$ and the units are $\mathrm{kgm}^{-3} . \mathrm{C}_{a}$ is the specific heat of air at constant pressure, $C_{f}$ stands for the bulk transfer coefficient, given by the expression $C_{f}=0.01\left(1 .+0.3 / W_{a f}\right)$, with $W_{a f}$ being the wind speed at the interface air/foliage. Here $W_{a f}=2 m s^{-1} . T_{a}$ represents the ambient temperature, while $T_{v}$ is the temperature of the vegetation cover. In (2) there are also the emissivities of the vegetation cover, $\epsilon_{v}$, and the soil $\epsilon_{S}$. The emissivity represents the ability of a body to emit energy, and ranges from 0 to 1 . The parameter $\sigma_{v}$ represents the fractional vegetation cover, which is taken here as $\sigma_{v}=1 .-\exp (-0.75 L A I)$ for grasses as given in [16]. The absorbed solar radiation is taken in this work as $R_{a v}=Q\left|\cos \left(\frac{\pi t}{12}\right)-\sin \left(\frac{\pi t}{12}\right)\right| \beta_{v}$, where $Q$ is the solar constant and $\beta_{v}$ is the coalbedo (fraction of absorbed energy). In [12] there is a study of the stationary solutions of a global climate model for different values of the solar constant $Q$.

The latent heat flux is given by

$$
L_{v}=L A I \rho_{a f} C_{f} l_{v} W_{a f}
$$


where $l_{v}$ is the latent heat of vaporization which, according to Henderson-Sellers [17], is given by

$$
l_{v}=1.91846 \times 10^{6}\left(\frac{T_{v}}{T_{v}-33.91}\right)^{2} \mathrm{kJkg}^{-1} .
$$

As for the equation for the evolution of temperature in 2D coordinates, it is given by

$$
\frac{\partial \gamma\left(T_{s}\right)}{\partial t}-\frac{\partial}{\partial x}\left(K_{H} \frac{\partial T_{s}}{\partial x}\right)-\frac{\partial}{\partial z}\left(K_{V} \frac{\partial T_{s}}{\partial z}\right)=F_{s}
$$

where $K_{H}$ and $K_{V}$ are the heat conductivities of the soil in $x$ and $z$ directions respectively. The coordinate $x$ is along the surface and $z$ represents depth. The equation (6) considers the water phase change, which is described by $\gamma\left(T_{S}\right)$. We notice that, in this model, heat capacity is not a constant, but a function of temperature. In this work it is introduced a similar expression to the one used in global climate models for latent heat of fusion, see [13], which is also similar to an expression utilised in green roof modelling for heat capacity, see [9], reading

$$
\gamma\left(T_{s}\right)=\left\{\begin{array}{clc}
k_{2} \tilde{T}_{s} & \text { if } & T_{s}<273 \\
\frac{L+k_{1} \epsilon_{0}}{\epsilon_{0}} \tilde{T}_{S} & \text { if } & T_{s} \in\left[273,273+\epsilon_{0}\right] \\
L+k_{1} \tilde{T}_{S} & \text { if } & T_{s}>273+\epsilon_{0}
\end{array}\right.
$$

where $\tilde{T}_{S}=T_{S}-273$. When solving the model (6) the heat capacity $\gamma\left(T_{S}\right)$ is obtained. According to the expression given in (7), the temperature of the soil, $T_{S}$, can be computed applying a solver of nonlinear equations. In this work we have implemented in the computer code a numerical technique based on an efficient combination of a Newton-Raphson's technique and another technique such as the bisection method or the regula falsi one. This process is automatized in the sense that the Newton-Raphson's technique is allowed to perform a certain number of iterations and, in the case it fails to converge, the other technique (bisection or regula falsi) starts automatically. This way to proceed is useful, since it is well-known that Newton-Raphson's method is the one that converge faster, in the vicinity of the sought solution, that is in local convergence, whilst bisection technique works in most of the cases. However, since bisection and regula falsi techniques require more iterations than Newton-Raphson's method, it is always advisable to start with this latter one. In order to illustrate the iterations carried out by the nonlinear solver in Figure 2 it is displayed the number of iterations carried out for a $2 D$ spatial mesh $50 \times 50=2500$ cells using a combination of Newton-Raphson's method with bisection and regula falsi ones attained in the numerical resolution. These results come from one of the numerical examples conducted in the next sections, but it is included in this one for the sake of the explanation of nonlinear solver. We recall that this is an automatic process, in the sense that regula falsi or bisection technique only intervenes when Newton-Raphson does not converges.

In this work the values $k_{1}=0.5, k_{2}=0.5, \epsilon_{0}=0.01, L=300$ are used.

In order to consider that the interaction between the vegetation cover and the soil takes place within the mixed layer, the right hand side of equation (6) is given by

$$
F_{s}\left(x, z, t, T_{s}, T_{v}\right)=\left\{\begin{array}{ccc}
f_{s, 1}-f_{s, 2}+H_{s}+L_{s} & \text { if } & z \leq D \text { mix } \\
0 & \text { otherwise, }
\end{array}\right.
$$

where, as aforementioned, Dmix is the depth of the mixed layer, where the influence of the vegetation on the soil takes place. In (6) $f_{s, 1}$ and $f_{s, 2}$ are defined making use of Stefan-Boltzmann's law for radiation and are expressed as

$$
\begin{gathered}
f_{s, 1}=\left(1-\sigma_{v}\right)\left(E-\epsilon_{s} \sigma T s^{4}\right), \\
f_{s, 2}=\frac{\sigma_{v} \epsilon_{v} \epsilon_{s} \sigma}{\epsilon_{l}}\left(T_{s}^{4}-T_{v}^{4}\right) .
\end{gathered}
$$

In the expression (9) it is introduced $E=R_{a s}+\epsilon_{s} I_{i r}$ where $R_{a s}$ is the radiation absorbed by the soil given by $R_{a s}=Q \mid\left(\sin \left(\frac{\pi t}{12}\right)+\cos \left(\frac{\pi t}{12}\right) \mid \beta_{s}\right.$ where $\beta_{s}$ is the coalbedo of the soil. 

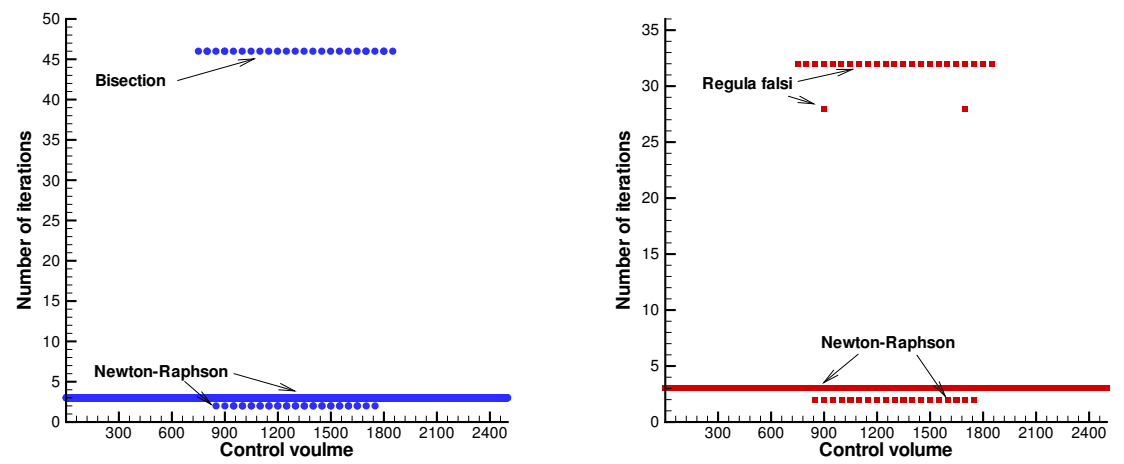

Figure 2. Number of iterations carried out for a spatial mesh $50 \times 50=2500$ cells using a combination of Newton-Raphson's method with bisection and regula falsi ones.

In (8) the following quantities are introduced

$$
H_{s}=\left(e_{0}+\rho_{a g} C_{a} C_{h g} W_{a f}\right)\left(T_{a}-T_{s}\right),
$$

and

$$
L_{s}=C_{e g} l_{v} W_{a f} \rho_{a g}\left(q_{a f}-q_{g}\right),
$$

where $q_{a f}=\frac{\left(1-\sigma_{v}\right) q_{a}+\sigma_{v}\left(0.3 q_{a}+0.6 q_{f s a t} r \text { seg }+0.1 q_{g s a t} M_{g}\right)}{1-\sigma_{v}\left(0.6(1-r s e g)+0.1\left(1-M_{g}\right)\right)}$ with $r s e g=\frac{r_{a}}{r_{a}+r_{s}}$, where $r_{a}=\frac{1}{C_{f} W_{a f}}$ is the resistance to moisture exchange and $r_{s}$ is the stomatal resistance.

We also consider the Bulk Richardson number given by $R_{i b}=\frac{2 g Z_{a}\left(T_{a f}-T s\right)}{\left(T_{a f}+T s\right) W_{a f}^{2}}$. According to this value it is defined the so-called atmospheric stability factor as

$$
\Gamma_{h}=\left\{\begin{array}{cll}
\frac{1}{\sqrt{1-16 R_{i b}}} & \text { if } & R_{i b}<0 \\
\frac{1}{1-5 R_{i b}} & \text { if } & R_{i b} \geq 0 .
\end{array}\right.
$$

We have $C_{h g}=\Gamma_{h}\left(\left(1-\sigma_{v}\right) C_{h n g}+\sigma_{v} C_{h n f}\right)$ with $C_{h n g}=\left(\frac{\kappa}{\log \left(Z_{a} / z_{o g}\right)}\right)^{2}$ and $C_{h n f}=\left(\frac{\kappa}{\log \left(Z_{a}-Z_{d} / z_{o f}\right)}\right)^{2}$ where $\kappa$ is the universal von Kármán constant, which is frequently used in turbulence modelling, as it happens in boundary-layer meteorology to account for fluxes of momentum, heat and moisture from the atmosphere to the land surface. We also have the values $C_{e g}=\Gamma_{h}\left(\left(1-\sigma_{v}\right) C_{e n g}+\sigma_{v} C_{h n f}\right)$ and $q_{g}=M_{g} q_{g s a t}+\left(1-M_{g}\right) q_{a f}$

The values taken in this work for the different parameters involved in this model for the numerical simulations are displayed in Table 1.

\section{Numerical approach}

In this section it is briefly described the numerical technique followed, which is developed in the finite volume framework. The main reasons behind using finite volume schemes are:

- They are conservative by construction in the sense that physical variables are conserved.

- Due to their discontinuity nature, since it makes use of cell averages of the solution, discontinuities are well resolved.

In this work a semi-discrete finite volume scheme is built for the vegetation cover, equation (1), and for the soil, equation (6). Regarding the vegetation cover (1), the $1 D$ domain $[-l, l]$ is discretized in $N_{x}$ control volumes. The equation (1) is integrated over each control volume dividing by its length. Let us 


\begin{tabular}{|c|c|c|}
\hline Density of the vegetation cover & $\rho_{v}$ & 1 \\
\hline Heat capacity of the vegetation cover & $C_{v}$ & 1 \\
\hline Density of the soil & $\rho_{S}$ & 1.21 \\
\hline Heat capacity of the air & $C_{a}$ & 1.1 \\
\hline Absorbed long wave radiation & $I_{i r}$ & 0.004 \\
\hline Emissivity of the vegetation & $\epsilon_{v}$ & 0.9 \\
\hline Emissivity of the soil & $\epsilon_{S}$ & 0.95 \\
\hline Emissivity & $\epsilon_{l}$ & 1 \\
\hline Leaf Area Index & $L A I$ & 5 \\
\hline Latent heat soil & $L_{S}$ & 2 \\
\hline Turbulent Schmidt number & $r_{c h}$ & 0.63 \\
\hline von Kármán constant & $\kappa$ & 0.4 \\
\hline Wind velocity & $W_{a f}$ & 2 \\
\hline Instrument height & $Z_{a}$ & 20 \\
\hline Gravity constant $\left(\mathrm{ms}^{-1}\right)$ & $g$ & 9.81 \\
\hline Ambient temperature $(K)$ & $T_{a}$ & 298 \\
\hline Foliage roughness & $z_{o f}$ & 0.03 \\
\hline Ground roughness & $z_{o g}$ & 0.02 \\
\hline Stefan-Boltzmann's constant $\left(\mathrm{Wm}^{-2} \mathrm{~K}^{-4}\right)$ & $\sigma$ & $5.67 \times 10^{-8}$ \\
\hline coalbedo vegetation & $\beta_{v}$ & 0.70 \\
\hline coalbedo soil & $\beta_{S}$ & 0.25 \\
\hline Solar constant & $Q$ & 340 \\
\hline Windless exchange coeff for sensible heat & $e_{0}$ & 2 \\
\hline Bulk transfer coeff & $Z_{d}$ & 0.5 \\
\hline Ratio of volumetric moisture & $M_{g}$ & 0.5 \\
\hline
\end{tabular}

Table 1. Values of the parameters used in the simulations

consider the control volume $S_{i}=\left[x_{i-\frac{1}{2}}, x_{i+\frac{1}{2}}\right]$ whose size is $\Delta x_{i}=x_{i+\frac{1}{2}}-x_{i-\frac{1}{2}}$ and integrate equation (1) on $S_{i}$ dividing by $\Delta x_{i}$ to yield

$$
\frac{d T_{v, i}(t)}{d t}=\frac{1}{\Delta x_{i}}\left(f_{i+\frac{1}{2}}-f_{i-\frac{1}{2}}\right)+F_{v, i}(t) \equiv l_{i}(t),
$$

being

$$
T_{v, i}(t)=\frac{1}{\Delta x_{i}} \int_{x_{i-\frac{1}{2}}}^{x_{i+\frac{1}{2}}} T_{v}(x, t) d x
$$

the spatial cell average of the temperature $T_{v}(x, t)$ in the control volume $S_{i}$ at time $t$,

$$
f_{i+\frac{1}{2}}=f\left(x_{i+\frac{1}{2}}, \frac{\partial T_{v}}{\partial x}\left(x_{i+\frac{1}{2}}, t\right)\right)
$$

is the right interface numerical flux at time $t$, and

$$
F_{v, i}(t)=\frac{1}{\Delta x_{i}} \int_{x_{i-\frac{1}{2}}}^{x_{i+\frac{1}{2}}}\left(F_{v}\left(x, T_{v}, T_{\mathcal{S}}\right)-K_{V} \frac{\partial T_{s}}{\partial z}\right) d x
$$

is the spatial average of the source term $F_{v}$ in the control volume $S_{i}$ at the particular time $t$.

Considering the soil equation (6), the $2 D$ region $[-l, l] \times[-H, 0]$ is discretized in $N_{x} \times N_{z}$ rectangular control volumes. Denoting as $I_{i, j}$ one of these $2 D$ control volumes of dimensions $\Delta x_{i} \times \Delta z_{j}$ where $\Delta x_{i}=x_{i+\frac{1}{2}}-x_{i-\frac{1}{2}}$ and $\Delta z_{j}=z_{j+\frac{1}{2}}-z_{j-\frac{1}{2}}$, integration on the control volume gives

$$
\frac{d \gamma_{i, j}(t)}{d t}=\frac{1}{\Delta x_{i}}\left(F_{i+\frac{1}{2}, j}-F_{i-\frac{1}{2}, j}\right)+\frac{1}{\Delta z_{j}}\left(G_{i, j+\frac{1}{2}}-G_{i, j-\frac{1}{2}}\right)+\Gamma_{i j} \equiv L_{i j}(t),
$$


where

$$
\gamma_{i, j}(t)=\frac{1}{\Delta x_{i} \Delta z_{j}} \int_{x_{i-\frac{1}{2}}}^{x_{i+\frac{1}{2}}} \int_{z_{j-\frac{1}{2}}}^{z_{j+\frac{1}{2}}} \gamma(x, z, t) d z d x,
$$

is the cell average of the soil temperature inside the control volume $I_{i j}$, while the value $F_{i+\frac{1}{2}, j}$ is the right intercell numerical flux in $x$-direction and $G_{i, j+\frac{1}{2}}$ is the intercell numerical flux in $z$-direction at time $t$, and

$$
\begin{gathered}
F_{i+\frac{1}{2}, j}=\frac{1}{\Delta z_{j}} \int_{z_{j-\frac{1}{2}}}^{z_{j+\frac{1}{2}}} F\left(x_{i+\frac{1}{2}}, \frac{\partial T_{s}}{\partial z}\left(x_{i+\frac{1}{2}}, z, t\right)\right) d z, \\
G_{i, j+\frac{1}{2}}=\frac{1}{\Delta x_{i}} \int_{x_{i-\frac{1}{2}}}^{x_{i+\frac{1}{2}}} G\left(T_{s}\left(x, z_{j+\frac{1}{2}}, t\right), \frac{\partial T_{s}}{\partial z}\left(x, z_{j+\frac{1}{2}}, t\right)\right) d x,
\end{gathered}
$$

are the spatial average of physical fluxes over cell faces at time $t$ and

$$
F_{s, i j}=\frac{1}{\Delta x_{i} \Delta z_{j}} \int_{x_{i-\frac{1}{2}}}^{x_{i+\frac{1}{2}}} \int_{z_{j-\frac{1}{2}}}^{z_{j+\frac{1}{2}}} F_{S}\left(x, z, T_{S}(x, z, t), T_{v}(x, z, t)\right) d z d x,
$$

is the spatial average of the source term $F_{S}\left(T_{S}\right)$ over the control volume $I_{i j}$.

The numerical solution may be evolved in time by means of different procedures. In this work it is performed a third order TVD Runge-Kutta method, as introduced in [18] and described in [12] for global climate modelling. The expressions of this method read

$$
\begin{aligned}
& \eta^{k, 1}=\eta^{n}+\Delta t \Lambda\left(\eta^{n}\right) \\
& \eta^{k, 2}=\frac{3}{4} \eta^{n}+\frac{1}{4} \eta^{k, 1}+\frac{1}{4} \Delta t \Lambda\left(\eta^{k, 1}\right), \\
& \eta^{k+1}=\frac{1}{3} \eta^{n}+\frac{2}{3} \eta^{k, 2}+\frac{2}{3} \Delta t \Lambda\left(\eta^{k, 2}\right)
\end{aligned}
$$

where $\eta$ refers to the temperature of the vegetation cover, $T_{v}$ or to $\gamma\left(T_{S}\right)$ for the evolution of the temperature in the soil. Furthermore, the operator $\Lambda(\cdot)$ is $l_{i}(\cdot)$ in (13) for the vegetation cover whereas the operator $L_{i j}(\cdot)$ in (17) for the soil part.

The main features of this process are:

- High-order reconstruction of fluxes at cell interfaces, achieved using Weighted Essentially Non Oscillatory (WENO) reconstruction.

- Third-order evolution in time, using a RK3-TVD approach.

For each particular time step, we first solve the evolution of the temperature in the $1 D$ vegetation cover, $T_{v}$, given the equation (1) and use this numerical solution as Dirichlet boundary condition to solve the equation (6) to compute the soil temperature $T_{s}$.

\subsection{Spatial WENO Reconstruction}

In a finite volume scheme, it is necessary to carry out a reconstruction process since, at each particular time step, a piecewise constant function is achieved, due to the use of cell averages of the solution. There are many ways to carry out this reconstruction process. Among them, Weighted Essentially Non Oscillatory (WENO) approach is used here. This is a nonlinear process, since weights depend on the solution itself. This nonlinear feature is important as, according to Godunov's theorem [19], there are no linear monotone numerical schemes of order higher than one. Due to this, nonlinear numerical schemes were introduced, aimed to circunvent Godunov's theorem and achieve monotone numerical schemes of order higher than one. Essentially Non Oscillatory (ENO) schemes, developed by Ami Harten and collaborators in 1980's, [20,21], were among the first successful relevant nonlinear numerical schemes which preserved monotonicity and high order of accuracy. WENO schemes were subsequently introduced, in which weights are assigned to each particular candidate stencil, taking into consideration certain smoothness indicators. In this work we utilise entire polynomials, see for instance [12,22-24], instead of getting pointwise values as in the classical WENO approach introduced in [25]. 
The use of entire polynomials allows to attain point values were needed, such as intercell boundaries or quadrature points. For the $2 D$ case we make use here of a dimension-by-dimension reconstruction procedure as detailed in [12,26-28] just to name a few of them. This process is less expensive than the fully $2 D$ WENO procedure. We note that there are other variants of WENO procedure that can be mentioned, such as CWENO (Central WENO approach) as described in in [29,30], the recently introduced CWENOZ scheme [31] where boundary conditions are introduced without using ghost cells, or WENO scheme with Unconditionally Optimal Accuracy as reported in [32].

In the $2 D$ dimension-by-dimension WENO procedure we consider the control volume $I_{i, j}$, where cell averages of the solution are computed, and introduce the following set of one-dimensional reconstruction stencils

$$
\mathcal{S}_{i, j}^{s, x}=\bigcup_{e=i-L}^{i+R} I_{e, j}, \quad \mathcal{S}_{i, j}^{s, z}=\bigcup_{e=j-L}^{j+R} I_{i, e}
$$

where $L$ and $R$ represent left and right spatial extension of the stencil. The process considered here follows the strategy put forward in different references such as [12,26,27], where three candidate stencils are used for odd order while four possible stencils are taken into account for the even order case. See the aforementioned references for details on this process.

We introduce the mapping $\left[x_{i}, x_{i+1}\right] \times\left[z_{j}, z_{j+1}\right] \rightarrow[0,1] \times[0,1]$ with $x=x_{i-\frac{1}{2}}+\xi \Delta x_{i}, z=$ $z_{j-\frac{1}{2}}+\eta \Delta z_{j}$, with $(\xi, \eta) \in[0,1]$. The way to perform, which is described in detail in several references, see, for instance [26], is briefly explained here.

We start by carrying out reconstruction in $x$-direction. For each control volume the reconstruction polynomials are

$$
w_{h}^{s, x}\left(x, t^{n}\right)=\sum_{p=0}^{\mathrm{M}} \psi_{p}(\xi) \hat{w}_{i j, p}^{n, s} \quad, \forall S_{i j}^{s, x},
$$

with the basis interpolation functions $\psi_{p}$, which in this work are Lagrange ones, although different types, such as Legendre ones, could also be used perfectly well. The values $\hat{w}_{i j, p}^{n, s}$ are the coefficients of interpolation. Now we get

$$
\frac{1}{\Delta x_{e}} \int_{x_{e-1 / 2}}^{x_{e+1 / 2}} \sum_{p=0}^{\mathrm{M}} \psi_{p}(\xi(x)) \hat{w}_{i j, p}^{n, s} d x=\bar{u}_{e j}^{n}, \quad \forall I_{e j} \in \mathcal{S}_{i j}^{s, x}
$$

applying integral conservation. If we carry out now a data-dependent nonlinear combination of the coefficients of the polynomials for each particular stencil it is obtained. Applying now a nonlinear combination, typical of WENO approach, of the coefficients it is achieved

$$
w_{h}^{x}\left(x, t^{n}\right)=\sum_{p=0}^{\mathrm{M}} \psi_{p}(\xi) \hat{w}_{i j, p^{\prime}}^{n} \quad \text { with } \quad \hat{w}_{i j, p}^{n}=\sum_{s=1}^{N_{s}} \omega_{s} \hat{w}_{i j, p^{\prime}}^{n, s}
$$

with $\omega_{s}=\frac{\tilde{\omega}_{s}}{\sum_{k} \tilde{\omega}_{k}}$, with $\tilde{\omega}_{s}=\frac{\lambda_{s}}{\left(\sigma_{s}+\epsilon\right)^{v}}$, and $\epsilon$ introduced to avoid division by zero. In this work it is used $\epsilon=10^{-20}$ and the parameter $v$ is taken as $v=3$. The oscillation indicators are

$$
\sigma_{s}=\sum_{p=1}^{\mathrm{M}} \sum_{m=1}^{\mathrm{M}} \Omega_{p m} \hat{w}_{i j, p}^{n, s} \hat{w}_{i j, m^{\prime}}^{n, s}
$$

with the oscillation matrix

$$
\Omega_{p m}=\sum_{\alpha=1}^{M} \int_{0}^{1} \frac{\partial^{\alpha} \psi_{p}(\xi)}{\partial \xi^{\alpha}} \cdot \frac{\partial^{\alpha} \psi_{m}(\xi)}{\partial \xi^{\alpha}} d \xi
$$


The next step consists of reconstruction in $z$-direction, performing in a similar way to the previous case, but applying conservation to each particular degree of freedom $\hat{w}_{i j, p}^{n}$. Then we have

$$
w_{h}^{s, z}\left(x, z, t^{n}\right)=\sum_{q=0}^{\mathrm{M}} \sum_{p=0}^{\mathrm{M}} \psi_{p}(\xi) \psi_{q}(\eta) \hat{w}_{i j, p q}^{n, s}
$$

Integral conservation in $z$-direction is carried out for each particular degree of freedom in $x$-direction, for all the control volumes of the stencil in $z$-direction, that is

$$
\frac{1}{\Delta z_{e}} \int_{z_{e-1 / 2}}^{z_{e+1 / 2}} \sum_{q=0}^{\mathrm{M}} \psi_{q}(\eta(z)) \hat{w}_{i j, p q}^{n, s} d z=\hat{w}_{i e, p}^{n}, \quad \forall I_{i e} \in \mathcal{S}_{i j}^{s, z}
$$

where $\mathcal{S}_{i j}^{s, z}$ are the control volumes in $z$-direction.

$$
w_{h}^{z}\left(x, z, t^{n}\right)=\sum_{q=0}^{\mathrm{M}} \sum_{p=0}^{\mathrm{M}} \psi_{p}(\xi) \psi_{q}(\eta) \hat{w}_{i j, p q}^{n} w i t h \quad \hat{w}_{i j, p q}^{n}=\sum_{s=1}^{N_{s}} \omega_{s} \hat{w}_{i j, p q}^{n, s} .
$$

Eventually it is obtained for the control volume $I_{i j}$

$$
w_{i j}\left(\xi, \eta, t^{n}\right)=\sum_{k=1}^{\mathrm{M}+1} \sum_{l=1}^{\mathrm{M}+1} \hat{w}_{i j}^{k, l}\left(t^{n}\right) \psi_{k}(\xi) \psi_{l}(\eta)
$$

as the final reconstruction polynomial.

In the present work we are using a WENO7 approach, where four cells are used for each stencil $(r=4, \mathrm{M}=3)$ and the developed scheme is seventh order accurate in space. This way to proceed is computationally less expensive than the fully two-dimensional reconstruction.

\section{Numerical examples}

The mathematical model under study is a coupled model formed by a vegetation cover and soil underneath it, describing the evolution of temperature. The main processes and equations are described in Section 2. The system (33) represents the full coupled model.

$$
\left\{\begin{array}{cc}
C_{v} \frac{\partial T_{v}}{\partial t}-\frac{\partial}{\partial x}\left(K_{H 0} \frac{\partial T_{v}}{\partial x}\right)+K_{V} \frac{\partial T_{s}}{\partial n}=F_{v}, & x \in(-l, l), t>0 \\
\frac{\partial \gamma\left(T_{s}\right)}{\partial t}-\frac{\partial}{\partial x}\left(K_{H} \frac{\partial T_{s}}{\partial x}\right)-\frac{\partial}{\partial z}\left(K_{V} \frac{\partial T_{s}}{\partial z}\right)=F_{s},(x, z) \in(-l, l) \times(-H, 0), t>0 \\
\frac{\partial T_{v}}{\partial x}(-l, t)=\frac{\partial T_{v}}{\partial x}(l, t)=0, & t>0 \\
\frac{\partial T_{s}}{\partial x}(-l, z, t)=\frac{\partial T_{s}}{\partial x}(l, z, t)=\frac{\partial T_{s}}{\partial z}(x,-H, t)=0, & x \in(-l, l), t>0 \\
T_{S}(x, 0, t)=T_{v}(x, t) ; & x \in(-l, l), t>0 \\
T_{v}(x, 0)=C \cdot \exp \left(-80 \cdot \sin ^{2}\left(x^{4}\right)\right)+298, & x \in(-l, l) \\
T_{S}(x, z, 0)=C \cdot \exp \left(-80 \cdot \sin ^{2}\left(x^{4}\right)-80 z^{2}\right)+290, & (x, z) \in(-l, l) \times(-H, 0)
\end{array}\right.
$$

In the problem (33) $C$ is a constant and it is considered the spatial domain $(-l, l) \times(-H, 0)$ as local reference coordinates. The numerical simulation is conducted applying the finite volume numerical scheme RK3TVD with WENO technique for spatial reconstruction as described in Section 3. As previously detailed, the nonlinearity appearing due to the heat capacity, which incorporates the 
latent heat of fusion, is solved by a Newton-Raphson's numerical scheme combined with regula falsi technique. The spatial mesh is formed by $50 \times 50$ control volumes. The size of the time step is computed as $\Delta t=\min \left(C F L \times \frac{\Delta x^{2}}{K_{H}}, C F L \times \frac{\Delta x^{2}}{K_{H}}, C F L \times \frac{\Delta z^{2}}{K_{V}}\right)$ where $C F L=0.35$ for stability reasons. Concerning the heat conduction we take $K_{H 0}=0.01, K_{H}=0.03$ and $K_{V}=0.03$.

In Figure 3 results for the evolution of the temperature in the vegetation layer are shown for time $t=0.5$. It has been taken $C=-17$ in the initial condition of the model (33) and an ambient temperature of $T_{a}=273+\sin (t)$. The curves displayed are labeled as: Without coupling, which means that $\frac{\partial T_{s}}{\partial n}=0$ in (1) and With coupling which means that $\frac{\partial T_{s}}{\partial n} \neq 0$. It can be observed that, in the non-coupled situation the temperature in the vegetation layer exhibits a smoother profile than in the coupled one, due to the effect of the soil, which can be considered as a major storage of heat, see [2], which may have an important influence on the vegetation cover. We study now the space-time

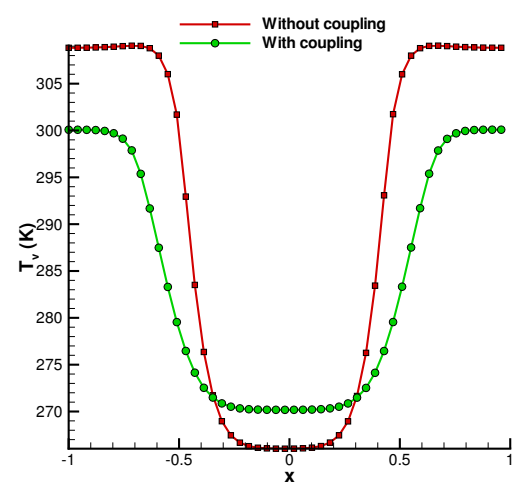

Figure 3. Temperature at the vegetation layer $\left(T_{v}\right)$. Output time $t=0.5$. With $C=-17$ and $T_{a}=$ $273+\sin (t)$. Without coupling means that $\frac{\partial T_{s}}{\partial n}=0$ in (1) while With coupling which means that $\frac{\partial T_{s}}{\partial n} \neq 0$.

evolution within the ground. The plots displayed in Figure 4 show the distribution of temperature $T_{S}$ inside the ground solving the problem (33) for several output times. It can be observed that the strongest variations of temperature take place in the mixed layer, close to the surface, whereas when advancing in depth, the temperature tends to an approximate constant value, which agrees with practical experience. In this case it has been taken again the value $C=6$ in the initial condition of the problem and the ambient temperature is $T_{a}=290+\sin (t)$. In this example and the subsequent ones, it has been taken $\frac{\partial T_{s}}{\partial n} \neq 0$ in the vegetation cover equation.

In Figure 5 it is depicted the evolution of the temperature $T_{s}$ with depth for different ambient temperatures. The results show that as depth increases the temperature attains a value close to the constant $298 \mathrm{~K}$, independently of the ambient temperature considered. This means that seasonal variations in the ambient temperature do not have an effect on the temperature of the soil, when depth increases, since the temperature remains close to a constant value. This fact has been verified in all the numerical examples presented. It also agrees with [33] where it is also stated that a similar situation takes place universally around the world.

The results displayed represent the spatial distribution of the temperature $T_{s}(0, z, 0.5)$, that is cutting the $2 D$ plots with $x=0$.

The last example considered takes into account a colder situation than the previous ones, taking $C=-40$ in the initial condition of the system (33) and, as ambient temperature, $T_{a}=265+\sin (t)$. Since the temperature ranges from values smaller than $273 \mathrm{~K}$ to values higher than $273 \mathrm{~K}$ the effect of the latent heat of fusion, that is present in the heat capacity of the soil. This is clearly visible in the top-left panel of Figure 6, where when the temperature in the soil is around $273 \mathrm{~K}$ the temperature remains approximately constant since the energy goes to the phase change, instead of to increase the temperature. This effect has also been studied in climate models, such as reported in [11]. In the top-right panel, the effect of latent heat of fusion is not considered. This means that it is used 

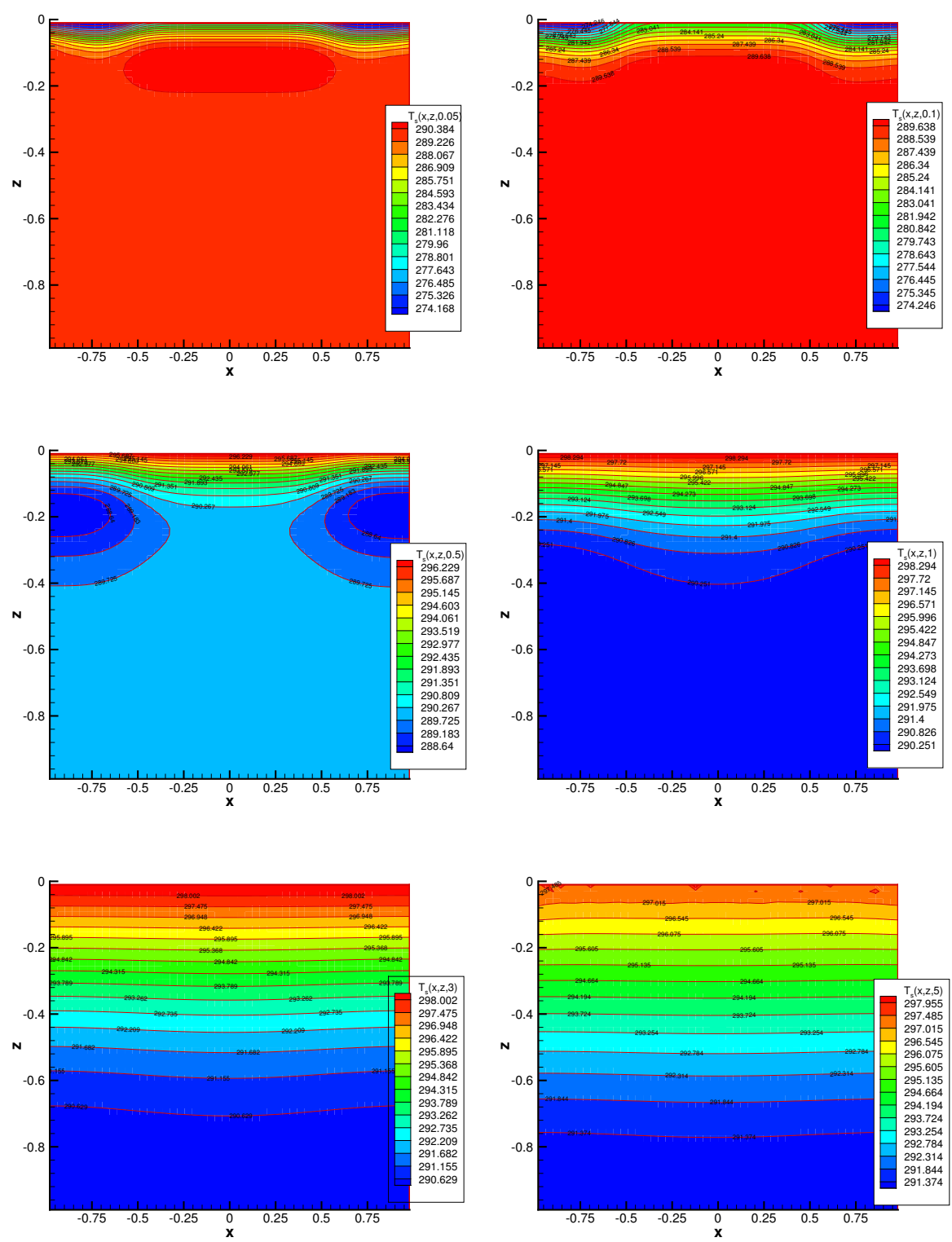

Figure 4. temperature in the soil $\left(T_{S}\right)$ for output times $=0.05,0.1,0.5,1,3,5$ when the ambient temperature is $T_{a}=290+\sin (t)$ and $C=6$ in the initial condition of (33). The depth is represented by the coordinate $z$.

$\gamma\left(T_{s}\right)=T_{s}$ in equation (33) and the plot shows that the variation of temperature is smoother than when latent heat of fusion is taken into consideration.

The bottom panel represents a cut with $x=0$ of the plots above for a more clear comparison of the cases with and without latent heat of fusion.

\section{Conclusions and Discussion}

In this work it is introduced a mathematical model describing the interaction between a $1 D$ vegetation cover and the $2 D$ ground underneath this cover. The model is coupled, in the sense that vegetation cover is a dynamic and diffusive boundary condition for the $2 D$ domain, it considers a mixed layer, where the heat exchange between both layers are taken into consideration and the equation of the vegetation cover includes a normal derivative accounting for heat conduction from the soil to the vegetation. The model also takes into account the water phase change, which is described by 


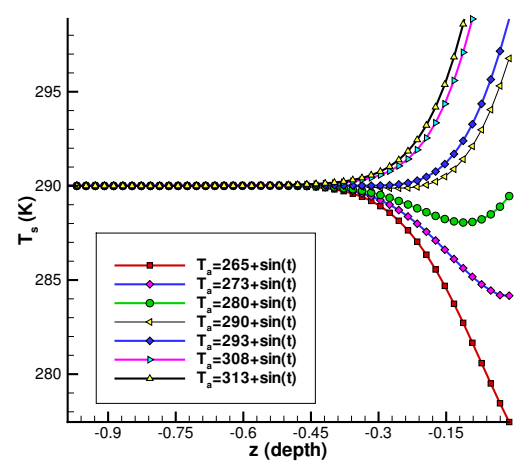

Figure 5. Soil temperature distribution with depth $\left(T_{S}(0 ., z, 0.5)\right)$.
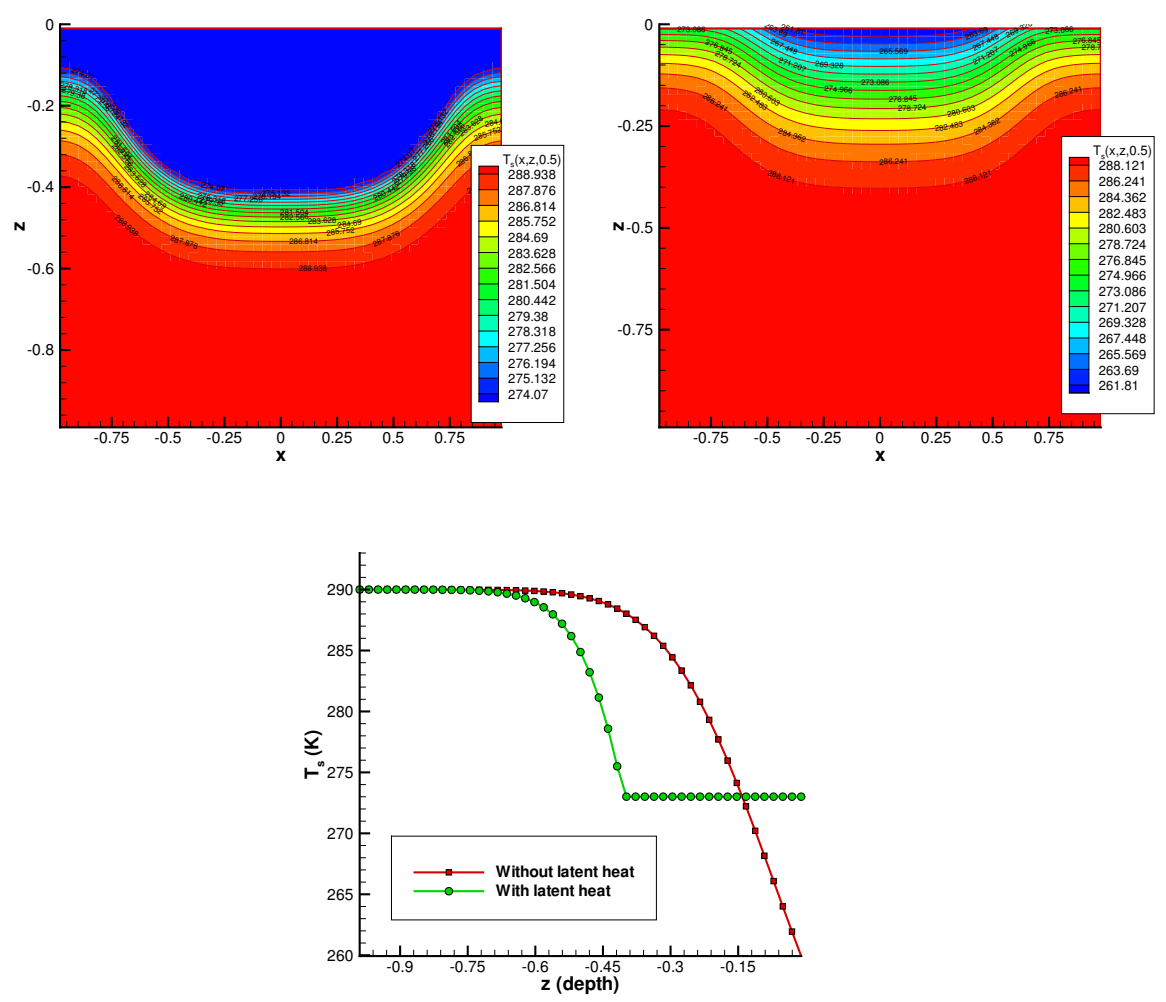

Figure 6. Temperature in the soil $\left(T_{s}\right)$ for output time $=0.5$ when the ambient temperature is $T_{a}=$ $265+\sin (t)$ and $C=-40$ in the initial condition of (33). The depth is represented by the coordinate $z$. Top: temperature in the soil considering latent heat (left panel) and without latent heat (right panel). Bottom: evolution of temperature in the soil $\left(T_{S}\right)$ cutting with $x=0$.

the function $\gamma\left(T_{S}\right)$, where $T_{S}$ is the temperature in the soil. Therefore, heat capacity is not a constant, but a function of temperature of the soil. This nonlinearity is resolved by means of a Newton-Raphson's technique combined with a regula falsi one (which acts automatically when Newton-Raphson's scheme fails to converge).

The main interactions between the vegetation and the soil take place in a thin region close to the surface, denoted here as mixed layer.

The mathematical model is solved using a finite volume numerical scheme, with a third order TVD Runge-Kutta scheme for time integration and a seventh order WENO approach for spatial reconstruction. These techniques are very efficient for solving this type of problems. 
The results obtained show that the main temperature variations take place in the vicinity of the surface, namely the mixed layer, whereas as depth increases the temperature tends to a constant value, as expected. This conclusion agrees with the real situation as reported in references on this topic.

Further research will include the consideration of water content and moisture in the soil, as well as taking into account different types of plants. In addition, it will be interesting to introduce the effect of precipitations, that may affect the integrity of the vegetation cover.

Author Contributions: Conceptualization, A.H. and L.T.; methodology, A.H. and L.T.; software, A.H. and L.T.; validation, A.H. and L.T.; formal analysis, A.H. and L.T.; investigation, A.H. and L.T.; resources, A.H. and L.T.; data curation, A.H. and L.T;; writing-original draft preparation, A.H. and L.T.; writing-review and editing, A.H. and L.T.; visualization, A.H. and L.T.; supervision, A.H. and L.T.; project administration, A.H. and L.T.; funding acquisition, A.H. and L.T. Both authors have read and agreed to the published version of the manuscript.

Funding: The research of both authors is partially supported by the projects MTM2017-85449-P Ministerio de Ciencia e Innovación, Spain and PID2020-112517GB-I00 Agencia Estatal de Investigación, Spain.

Conflicts of Interest: The authors declare no conflict of interest.

\section{Abbreviations}

The following abbreviations are used in this manuscript:

$\begin{array}{ll}\text { CAC } & \text { Cation Exchange Capacity } \\ \text { TVD } & \text { Total Variation Diminishing } \\ \text { WENO } & \text { Weighted Essentially Non Oscillatory } \\ \text { RK } & \text { Runge-Kutta } \\ \text { RK3-TVD } & \text { Third order Runge-Kutta TVD } \\ \text { FV } & \text { Finite Volume } \\ \text { PDE } & \text { Partial Differential Equation } \\ \text { CFL } & \text { Courant-Friedrichs-Lewy }\end{array}$

\section{References}

1. De Vries, D.A., Thermal properties of soils. In Physics of Plant Environment. Van Wijk, W. R., ed; North-Holland, Amsterdam, 1963; p. 210-235.

2. Onwuka, B.; Mang, B. Effects of soil temperature on some soil properties and plant growth. Adv Plants Agric Res. 2018, 8, 34-37. doi:10.15406/apar.2018.08.00288.

3. Yu, X.; Zhang, X.; Li, J.; Zhang, M.; Xie, Y. Effects of vegetation cover and precipitation on the process of sediment produced by erosion in a small watershed of loess region. Acta Ecologica Sinica 2006, 26, 1-8. doi:https://doi.org/10.1016/S1872-2032(06)60001-2.

4. Dondi, G.; Zhao, B.; Zhang, L.; Xia, Z.; Xu, W.; Xia, L.; Liang, Y.; Xia, D. Effects of Rainfall Intensity and Vegetation Cover on Erosion Characteristics of a Soil Containing Rock Fragments Slope. Advances in Civil Engineering, Hindawi 2019. doi:10.1155/2019/7043428.

5. Liu, J.; Gao, G.; Wang, S.; Jiao, L.; Wu, X.; Fu, B. The effects of vegetation on runoff and soil loss: Multidimensional structure analysis and scale characteristics. Journal of Geographical Sciences 2018, 28, 59-78. doi:10.1007/s11442-018-1459-z.

6. Martin, C.A.; Proulx, R.; Vellend, M.; Fahrig, L. How the relationship between vegetation cover and land-cover variance constrains biodiversity in a human dominated world. Landscape Ecology 2021, 36, 3097-3104. doi:10.1007/s10980-021-01312-9.

7. Meron, E.; Bennett, J.J.R.; Fernandez-Oto, C.; Tzuk, O.; Zelnik, Y.R.; Grafi, G. Continuum Modeling of Discrete Plant Communities: Why Does It Work and Why Is It Advantageous? Mathematics 2019, 7. doi:10.3390/math7100987.

8. Lozano-Parra, J.; Pulido, M.; Lozano-Fondón, C.; Schnabel, S. How do Soil Moisture and Vegetation Covers Influence Soil Temperature in Drylands of Mediterranean Regions? Water 2018, 10. doi:10.3390/w10121747.

9. Tello, J.I.; Tello, L.; Vilar, M.L. On the Existence of Solutions of a Two-Layer Green Roof Mathematical Model. Mathematics 2020, 8. doi:10.3390/math8091608.

10. Vilar, M.; Tello, L.; Hidalgo, A.; Bedoya, C. An energy balance model of heterogeneous extensive green roofs. Energy and Buildings 2021, 250, 111265. doi:https://doi.org/10.1016/j.enbuild.2021.111265. 
11. Díaz J. I., Hidalgo A., Tello L.. Multiple solutions and numerical analysis to the dynamic and stationary models coupling a delayed energy balance model involving latent heat and discontinuous albedo with a deep ocean. Proc. R. Soc. A. 2014. doi:10.1098/rspa.2014.0376.

12. Hidalgo, A.; Tello, L. Numerical Approach of the Equilibrium Solutions of a Global Climate Model. Mathematics 2020, 8. doi:10.3390/math8091542.

13. A. Hidalgo, L. Tello and E.F. Toro. Numerical and analytical study of an atherosclerosis inflammatory disease model. Journal of Mathematical Biology 2014, 68, 1785-1814. doi:10.1007/s00285-013-0688-0.

14. Hidalgo, A.; Tello, L. Numerical simulation of a porous medium-type atherosclerosis initiation model. ComputersEFluids 2018, 169, 380 - 387. Recent progress in nonlinear numerical methods for time-dependent flow\& transport problems, doi:10.1016/j.compfluid.2017.07.019.

15. Frankenstein, S.; Koenig, G.G. Fast All-Season Soil STrength (FASST); ERDC/CRREL TR-04-25 2004, U.S. Army, 2004.

16. Ramirez, J.A.; Senarath, S.U. Statistical-dynamical parameterization of interception and land surface-atmosphere interactions. Journal of Climate 2000, 13, 4050-4063. doi:https://doi.org/10.1016/j.enbuild.2021.111265.

17. Henderson-Sellers, B. A new formula for latent heat of vaporization of water as a function of temperature. Quarterly Journal of the Royal Meteorological Society 1984, 110, 1186-1190. doi:https:/ / doi.org/10.1002/qj.49711046626.

18. Gottlieb, S.; Shu, C.W. Total Variation Diminishing Runge-Kutta schemes. Mathematics of Computation 1998, 67, 73-85. doi:10.1090/S0025-5718-98-00913-2.

19. Godunov, S. A Difference Scheme for Numerical Solution of Discontinuous Solution of Hydrodynamic Equations. Math. Sbornik 1959, 47, 271-306.

20. Harten, A. High resolution schemes for hyperbolic conservation laws. Journal of Computational Physics 1983, 49, 357 - 393. doi:https://doi.org/10.1016/0021-9991(83)90136-5.

21. Harten, A.; Engquist, B.; Osher, S.; Chakravarthy, S.R. Uniformly High Order Accurate Essentially Non-oscillatory Schemes, III. Journal of Computational Physics 1997, 131, 3 - 47. doi:https://doi.org/10.1006/jcph.1996.5632.

22. Dumbser, M.; Enaux, C.; Toro, E.F. Finite volume schemes of very high order of accuracy for stiff hyperbolic balance laws. Journal of Computational Physics 2008, 227, 3971 - 4001. doi:10.1016/j.jcp.2007.12.005.

23. Dumbser, M. Arbitrary high order PNPM schemes on unstructured meshes for the compressible Navier-Stokes equations. Computers \& Fluids 2010, 39, 60 - 76. doi:10.1016/j.compfluid.2009.07.003.

24. Dumbser, M.; Hidalgo, A.; Castro, M.; Parés, C.; Toro, E.F. FORCE schemes on unstructured meshes II: Non-conservative hyperbolic systems. Computer Methods in Applied Mechanics and Engineering 2010, 199, 625 - 647. doi:10.1016/j.cma.2009.10.016.

25. Jiang, G.S.; Shu, C.W. Efficient Implementation of Weighted ENO Schemes. Journal of Computational Physics 1996, 126, 202 - 228. doi:10.1006/jcph.1996.0130.

26. Dumbser, M.; Zanotti, O.; Hidalgo, A.; Balsara, D.S. ADER-WENO finite volume schemes with space-time adaptive mesh refinement. Journal of Computational Physics 2013, 248, 257 - 286. doi:10.1016/j.jcp.2013.04.017.

27. Dumbser, M.; Hidalgo, A.; Zanotti, O. High order space-time adaptive ADER-WENO finite volume schemes for non-conservative hyperbolic systems. Computer Methods in Applied Mechanics and Engineering 2014, 268, 359 - 387. doi:10.1016/j.cma.2013.09.022.

28. Titarev, V.; Toro, E. Finite-volume WENO schemes for three-dimensional conservation laws. Journal of Computational Physics 2004, 201, 238 - 260. doi:10.1016/j.jcp.2004.05.015.

29. Castro, M.J.; Semplice, M. Third- and fourth-order well-balanced schemes for the shallow water equations based on the CWENO reconstruction. Mathematical and Computer Modelling 2019, 89, 304 325. doi:10.1002/fld.4700.

30. Cravero, I.; Puppo, G.; Semplice, M.; Visconti, G. CWENO: Uniformly accurate reconstructions for balance laws. Math. Comp. 2018, 87, 1689-1719. doi:10.1090/mcom/3273.

31. Semplice, M.; Travaglia, E.; Puppo, G. One- and Multi-dimensional CWENOZ Reconstructions for Implementing Boundary Conditions Without Ghost Cells. Communications on Applied Mathematics and Computation 2021, 17, 609 - 618. doi:10.1007/s42967-021-00151-4. 
32. Baeza, A.; Bürger, R.; Mulet, P.; Zorío, D. An Efficient Third-Order WENO Scheme with Unconditionally Optimal Accuracy. SIAM J. Sci. Comput. 2020, 42, A1028-A1051. doi:10.1137/19M1260396.

33. Selker, John and Or, Dani. Soil Hydrology and Biophysics; Oregon State University. 